\title{
Synergistic Eeffect of Polyene Antifungals and Silver Nanoparticles Against Candida Parapsilosis
}

\author{
da Frota $\mathrm{SM}^{1,4}$, Cunha FA ${ }^{* 1,5}$, Cunha $\mathrm{MDCDSO}^{3}$, Martins $\mathrm{RT}^{2}$, Menezes $\mathrm{EA}^{1}$, and Fechine $\mathrm{PBA}^{5}$ \\ ${ }^{1}$ Department of Clinical Analysis, Laboratory of Microbiology of Yeasts, Federal University of Ceará , UFC, Brazil \\ ${ }^{2}$ Laboratório de Análises Clínicas- Universidade da Federal do Ceará, Brazil \\ ${ }^{3}$ Professora do Curso de Enfermagem da Faculdade Princesa do Oeste- Crateús- Ceará, Brazil \\ ${ }^{4}$ Renorbio- Rede Nordeste de Biotecnologia, Brazil \\ ${ }^{5}$ Advanced Materials Chemistry Group (GQMAT)- Department of Analytical and Physical Chemistry, Federal Uni- \\ versity of Ceara- UFC, Campus do Pici, Fortaleza-Ceará- Brazil
}

${ }^{*}$ Corresponding author: Cunha FA, Advanced Materials Chemistry Group (GQMAT) - Department of Analytical and Physical Chemistry Chemistry, Federal University of Ceará - UFC, Campus do Pici, CP 12100, CEP 60451-970 Fortaleza - CE, Brazil, E-mail: afranio@ufc.br

Citation: da Frota SM, Cunha FA, Cunha MDCDSO, Martins RT, Menezes EA, et al. (2018) Synergistic effect Synergistic Effect of Polyene Antifungals and Silver Nanoparticles against Candida Parapsilosis. J Antibio Res 2(1): 104

Received Date: April 24, 2018 Accepted Date: June 14, 2018 Published Date: June 15, 2018

\begin{abstract}
Candidiasis is a fungal infection caused by yeasts of the genus Candida spp. Among several pathogenic Candida species, we can highlight the C. parapsilosis due to susceptibility profile. Amphotericin B and nystatin are polyene antifungal drugs most frequently used to combat this type of infection. Silver nanoparticles (AgNPs) also have antifungal properties and can also provide synergistic action when combined with classic antifungals. Therefore, the aim of this study was evaluate the synergistic action of AgNPs, amphotericin B and nystatin against $C$. parapsilosis. The synthesis of AgNPs were performed with glucose, and sodium dodecyl sulfate (SDS) was used as a stabilizer. The characterization of AgNPs was performed by scanning electron microscopy (SEM) and atomic force microscopy (AFM). It was found a value of $18.17 \mathrm{~nm}( \pm 5.3)$ for the size of the AgNPs, when measured by AFM. AgNPs were shown to be quite uniform and stable, and when combined with amphotericin B and nystatin, showed potent antifungal activity and increased the zone around the antifungal disk by 222.6 and $319.3 \%$, respectively. These results contribute to a better understanding of the synergistic effect and offers another option for the treatment of fungal infections.
\end{abstract}

Keywords: C. parapsilosis; Silver Nanoparticles; Polyene Antifungals; Synergistic Effect

\section{Introduction}

Infections caused by yeasts of the genus Candida are highly relevant due to their high morbidity and mortality rates, which can be compared to septic shock [1,2]. Infections caused by Candida spp. include about $80 \%$ of all fungal infections reported in hospitals; blood, urine and surgical wounds are the main body sites involved. Candida parapsilosis is an yeast constantly isolated from candidemia and exhibits mortality rates that are considered high. Its identification is no simple task, requiring biochemical, phenotypic and molecular methods [1].

There are many types of drugs used to treat fungal infections, such as the polyene antibiotics, which are broad-spectrum antifungals such as nystatin and amphotericin B. The former is part of many topical preparations available on the market, and the latter is an intravenous drug, widely used in combating candidemia [3]. The continuous use of these antifungals may promote the selection of resistant pathogenic fungi, not controlled by the previously effective fungicide, thereby jeopardizing its efficacy. Therefore, several strategies can be used to prevent or reduce fungal resistance, and one of the most common ones recently is to evaluate the synergism of antifungal drugs with other substances: essential oils, formulations with other antibiotics, and drugs without antifungal indication [4-7].

Silver nanoparticles (AgNPs) are structures with sizes up to $100 \mathrm{~nm}$ and exhibit different properties than those found in the source material. AgNPs are unstable and their synthesis involves many products, sometimes toxic, as the preparation methods can be chemical, biological or physical $[8,9]$. This kind of material has emerged as a promising and potent treatment against microorganisms due to its antibacterial, antiviral and antifungal effects, which are described in several studies [10,11]. In this way, 
the purpose of this study was evaluate the synergistic action between two types of polyene antibiotics (nystatin and amphotericin B) associated with AgNPs against strains of C. parapsilosis isolated from candidemia.

\section{Materials and Methods}

\section{Origin of the strains}

Ten strains of C. parapsilosis isolated from candidemia of patients admitted to public hospitals in Fortaleza, Ceará, in Northeastern Brazil, were used in this study. These were identified by biochemical, phenotypic and molecular methods [12]. These yeasts belong to the collection of the Laboratory of Microbiology of the Faculty of Pharmacy of the Federal University of Ceará.

\section{Production of AgNPs}

The AgNPs were synthesized using a solution of $5 \mathrm{mM}$ silver nitrate (Dinâmica - São Paulo - Brazil), 1.0g glucose (Dinâmica - São Paulo - Brazil) as a reducing agent, and 0.5g sodium dodecyl sulfate (SDS) (Vetec - Rio de Janeiro - Brazil) as a stabilizer [13].

\section{Characterization of the AgNPs}

The morphology, distribution and size of the AgNPs were determined using Scanning Electron Microscopy (SEM) and atomic force microscopy (AFM).

\section{Evaluation of the synergism of amphotericin B and nystatin with AgNPs}

The synergism effect was evaluated by the disk diffusion method on Mueller-Hinton medium (Himedia- Mumbai -India) supplemented with $2 \%$ glucose and $0.5 \mu \mathrm{g} / \mathrm{mL}$ methylene blue. We used commercial discs containing amphotericin B 100 $\mu \mathrm{g}(\mathrm{AB})$ $\left(\right.$ Cecon $\left.^{\oplus}\right)$ (São Paulo- Brazil), and nystatin 100 IU (NY)- (Cecon ${ }^{\oplus}$ ) (São Paulo- Brazil). Each disc was impregnated with $10 \mu \mathrm{L}$ of AgNPs. The disks were labeled as Ag@AB (disk containing amphotericin B and AgNP); Ag@NY (disk containing nystatin and AgNP); AB (disk containing only amphotericin B) and NY (disk containing only nystatin). Four discs were placed in each plate with C. parapsilosis. The plates were incubated at $35^{\circ} \mathrm{C}$ for $24-48 \mathrm{~h}$ and strains were tested three times [14].

In this study, synergism was considered as an increase in the area around the antifungal discs impregnated with AgNPs. The following formula was used: $\left(B^{2}-A^{2}\right) / A^{2}$ and $\left(D^{2}-C^{2}\right) / C^{2}$, where $A, B, C$ and $D$ are the inhibition zones of the disc of amphotericin B (A), Ag@ANF (B), nystatin (C) and Ag@ NYS (D). Increases in area were considered as synergism; no change was seen as indifferent, and decrease was regarded as antagonism [14,15].

\section{Results}

Figure 1 shows the characterization of AgNPs used in this study. As can be seen in the SEM (Figure 1 (a)) and AFM (Figure 1 (c)) images, the nanoparticles are well distributed with no agglomerations. The average size found by SEM (Figure 1(b)) and AFM (Figure $1(\mathrm{~d})$ ) were $24.04 \mathrm{~nm}( \pm 17.93)$ and $18.17 \mathrm{~nm}( \pm 5.83)$, on average ( \pm standard deviation), respectively.
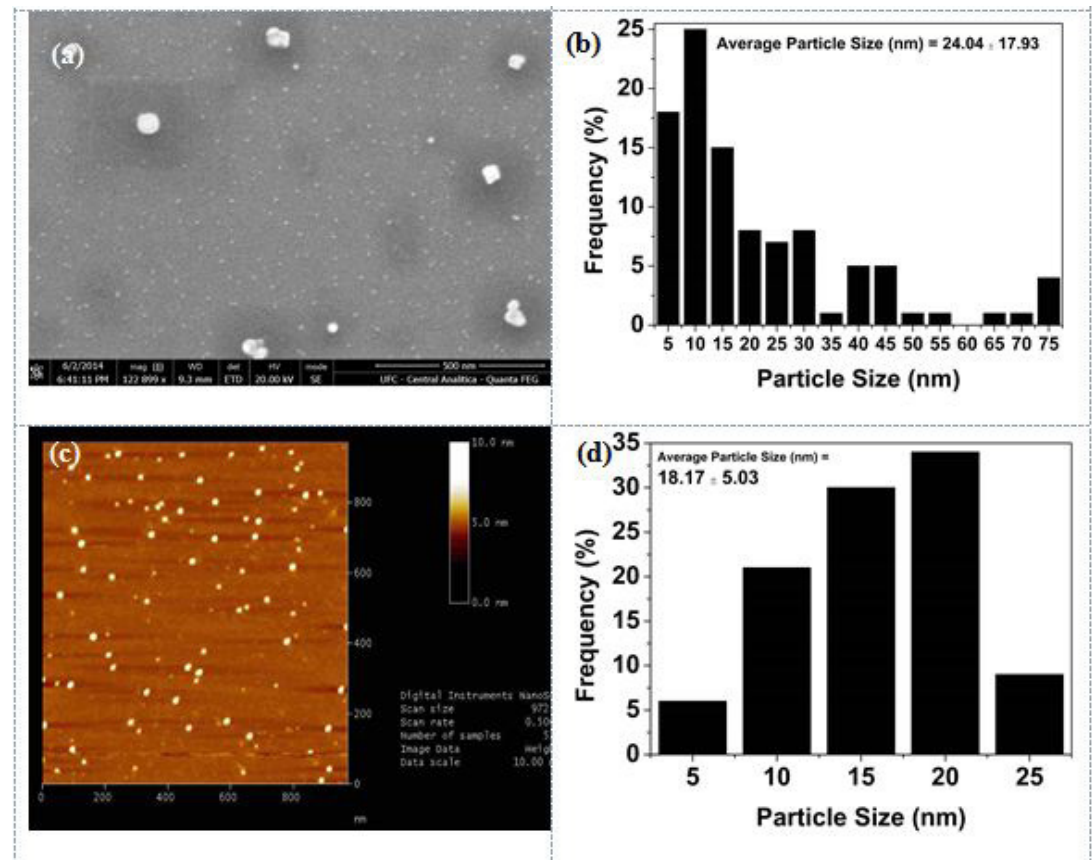

Figure 1: SEM (a), histogram obtained from SEM images (b), AFM (c) and histogram obtained from AFM images (d) of AgNPs 
The identification of C. parapsilosis was carried out in a chromogenic medium. In such medium, this yeast exhibits a characteristic pink color, as shown in Figure 2 (a). The identification was confirmed in micromorphology. Figure 2 (b) shows high synergistic activity with the combination of amphotericin B and nystatin with AgNPs.
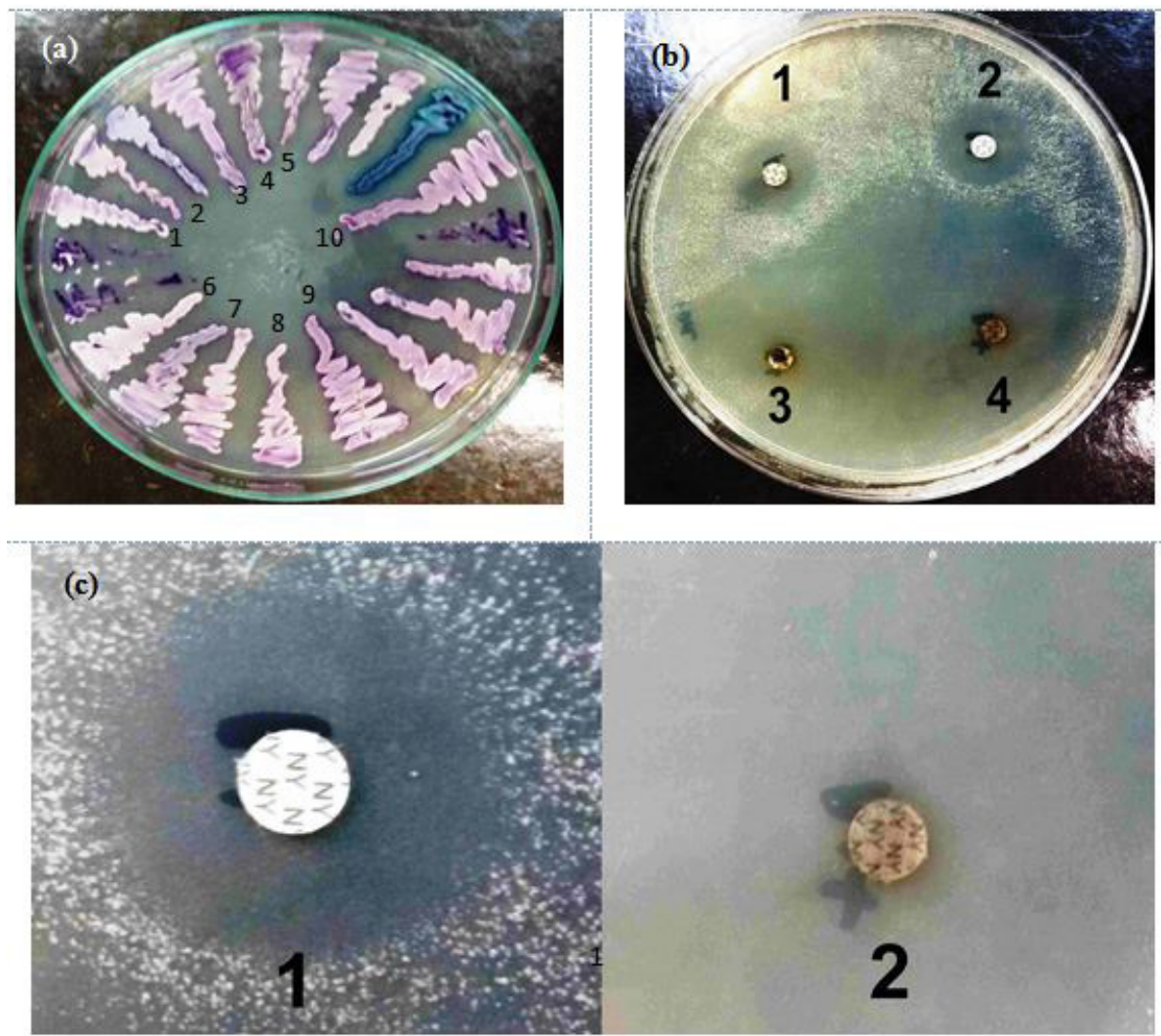

Figure 2: (a) C. parapsilosis on chromogenic medium (1-10). (b) Effect of polyene antifungals alone and associated with AgNPs: 1- amphotericin B alone; 2-nystatin alone; 3-Ag@AB; 4-Ag@NY. (c) Detail shows the effect of nystatin alone (1) and Ag@NY (2)

The disks impregnated with AgNPs showed an increased inhibition zone, which characterizes synergistic action. In Figure 2 (c), we can observe in detail the enlargement of the area around the nystatin disk (NY). This result can be observed numerically in Table 1 and, as can be seen in Figure 3, the combination of polyene antifungals and AgNPs were effective against C. parapsilosis and statistically significant (Student t-test). The enlargement of the area around the disks of antifungals impregnated with AgNPs was 222.6\% for Ag@AB and 319.3\% for Ag@ NY, when compared with discs containing only antifungal agents (Table 1).

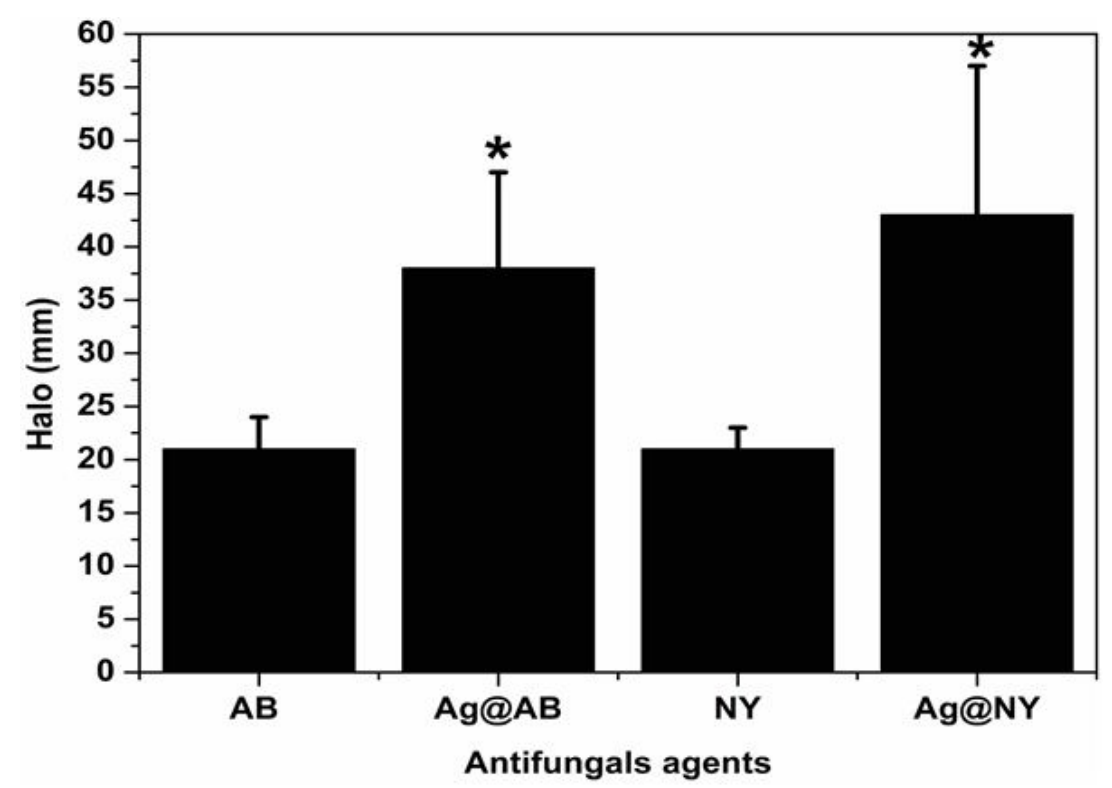

Figure 3: Effect of polyene antifungals alone and associated with AgNPs, AB- amphotericin B alone; NY-nystatin alone; 3-Ag@AB-Amphotericin B + AgNP; Ag@NY- Nystatin + AgNP. *Significantly different from antifungal discs $p<0.001$ 


\begin{tabular}{|c|c|c|c|c|c|c|}
\hline Yeasts(n) & \multicolumn{4}{|c|}{ Halo $(\mathbf{m m})$} & \multicolumn{2}{c|}{ Increased fold area (\%) } \\
\hline & $\mathbf{A}$ & $\mathbf{B}$ & $\mathbf{C}$ & $\mathbf{D}$ & $\left.\left(\mathbf{B}^{2}-\mathbf{A}^{2}\right) / \mathbf{A}^{2}\right)$ & $\left.\left(\mathbf{D}^{2}-\mathbf{C}^{2}\right) / \mathbf{C}^{2}\right)$ \\
\hline & $\mathrm{AB}$ & $\mathrm{Ag} @ \mathrm{AB}$ & $\mathrm{NY}$ & $\mathrm{Ag} \mathrm{NY}$ & $\mathrm{Ag} @ \mathrm{AB}$ & $\mathrm{Ag} @ \mathrm{NY}$ \\
\hline C. parapsilosis (10) & 21.1 & 37.9 & 21.0 & 43.0 & 222.6 & 319.3 \\
\hline
\end{tabular}

AB- amphotericin B; Ag@AB- amphotericin B +AgNP; NY- Nystatin, Ag@NY- Nystatin+AgNP. Mean surface area of the inhibition zone $\left(\mathrm{mm}^{2}\right)$ was calculated for each combination tested from the mean diameter. Increased fold area was calculated using $\left(\mathrm{B}^{2}-\mathrm{A}^{2}\right) / \mathrm{A}^{2}$ and $\left(\mathrm{D}^{2}-\mathrm{C}^{2}\right) / \mathrm{C}^{2}(\mathrm{x} 100)$, where $\mathrm{A}$ and $\mathrm{B}$ and $\mathrm{C}$ are the inhibition zones for A, B, C and D, respectively.

Table 1: Antifungal activity of AB, NY discs alone and Ag@AB, Ag@NY against C. parapsilosis

\section{Discussion}

Silver nanoparticles (AgNPs) can become a valuable ally in fighting resistant fungal infections [16]. The AgNPs produced in this study were shown to be stable and were obtained by a simple and inexpensive method (Figure 1).

In a study analyzing the synergistic action of AgNPs and antimicrobials against a group of bacteria, using the same method used in this study, the increase in area was evident, which demonstrates the potential of these compounds [14]. Studying the antifungal activity of AgNP and fluconazole, observed a powerful synergistic effect, primarily against C. albicans, analyzing the effect of the combination of AgNPs and nystatin against biofilms of C. albicans and C. glabrata, observed a synergistic effect and highlighted the importance of this combination $[15,17]$. Our results showed that the association of amphotericin B and nystatin with AgNPs was extremely effective against C. parapsilosis (Figure 2).

In a study of AgNPs and the antifungals fluconazole and itraconazole against $C$. albicans, a strong synergistic effect was observed [18]. However, our results were more expressive, since the studies described above used few microbial strains, whereas 10 strains of $C$. parapsilosis were used in our study. Polyene antifungals act by binding to the plasma membrane of $C$. parapsilosis, which leads to death [4]. AgNPs bind to important cell structures - proteins and DNA- and cause significant cellular damage [16]. The AgNPs and polyene antifungals act in different locations which enhancing the synergistic action. The combination of both substances can have their effects potentiated, therefore showing a large zone of growth inhibition (Figure 2 and 3). Therefore, the C. parapsilosis used in this study showed high sensitivity to polyene antifungals when combined with AgNPs.

\section{Conclusion}

In summary, our results showed that this new technological product (polyene antifungals and AgNPs) can help fight fungal infections. Other studies with a larger number of strains and toxicity tests should be conducted to know the potential of this combination.

\section{Acknowledgments}

The authors gratefully thank the Central Analítica-UFC/CT-INFRA/MCTI-SISNANO/Pró-Equipamentos, Departamento de Física da UFC, CAPES, CNPq and Funcap, for the grant provided to support the research on nanoparticles.

\section{References}

1. Colombo AL, Guimarães T, Camargo LF, Richtmann R, Queiroz-Telles Fd, et al. (2013) Brazilian guidelines for the management of candidiasis - a joint meeting report of three medical societies: Sociedade Brasileira de Infectologia, Sociedade Paulista de Infectologia and Sociedade Brasileira de Medicina Tropical. Braz J Infect Dis 17: 283-312.

2. Eggimann P, Pittet D (2010) Candidemia y candidiasis generalizada. EMC-Anestesia-Reanim 36: 1-26.

3. Denning DW, Hope WW (2010) Therapy for fungal diseases: opportunities and priorities. Trends Microbiol, 18: 195-204.

4. Amber K, Aijaz A, Immaculata X, Luqman KA, Nikhat M (2010) Anticandidal effect of Ocimum sanctum essential oil and its synergy with fluconazole and ketoconazole. Phytomedicine 17: 921-5.

5. Gao Y, Zhang C, Lu C, Liu P, Li Y, et al. (2013) Synergistic effect of doxycycline and fluconazole against Candida albicans biofilms and the impact of calcium channel blockers. FEMS Yeast Res 13: 453-62.

6. Zeidler U, Bougnoux ME, Lupan A, Helynck O, Doyen A, et al. (2013) Synergy of the antibiotic colistin with echinocandin antifungals in Candida species. J Antimicrob Chemother 68: 1285-96.

7. Menezes EA, Vasconcelos Júnior AA, Silva CL, Plutarco FX, Cunha Mda C, et al. (2012) In vitro synergism of simvastatin and fluconazole against Candida species. Rev Inst Med Trop Sao Paulo 54: 197-9.

8. Li G, He D, Qian Y, Guan B, Gao S, et al. (2012) Fungus-Mediated Green Synthesis of Silver Nanoparticles Using Aspergillus terreus. Int J Mol Sci 13: 466-76.

9. Nath D, Banerjee P (2013) Green nanotechnology - a new hope for medical biology. Environ Toxicol Pharmacol 36: 997-1014.

10. Bindhu M.R, Umadevi M (2015) Antibacterial and catalytic activities of green synthesized silver nanoparticles. Spectrochim Acta A Mol Biomol Spectrosc135: 373-8.

11. Qian Y, Yu H, He D, Yang H, Wang W, et al. (2013) Biosynthesis of silver nanoparticles by the endophytic fungus Epicoccum nigrum and their activity against pathogenic fungi. Bioprocess Biosyst Eng 36: 1613-9. 
12. de Vasconcelos AA Jr, Menezes EA, Cunha FA (2011) Chromogenic medium for direct susceptibility testing of Candida spp. isolated from urine. Mycopathologia 172: 125-30.

13. Darroudi M, Ahmad MB, Abdullah AH, Ibrahim NA (2011) Green synthesis and characterization of gelatin-based and sugar-reduced silver nanoparticles. Int J Nanomedicine 6: 569-74.

14. Birla SS, Tiwari VV, Gade AK, Ingle AP, Yadav AP, et al. (2009) Fabrication of silver nanoparticles by Phoma glomerata and its combined effect against Escherichia coli, Pseudomonas aeruginosa and Staphylococcus aureus. Lett Appl Microbiol 48: 173-9.

15. Monteiro DR1, Silva S, Negri M, Gorup LF, de Camargo ER, et al. (2013) Antifungal activity of silver nanoparticles in combination with nystatin and chlorhexidine digluconate against Candida albicans and Candida glabrata biofilms. Mycoses 56: 672-80.

16. Rai M, Yadav A, Gade A (2009) Silver nanoparticles as a new generation of antimicrobials. Biotechnol Adv 27: 76-83.

17. Gajbhiye M, Kesharwani J, Ingle A, Gade A, Rai M (2009) Fungus-mediated synthesis of silver nanoparticles and their activity against pathogenic fungi in combination with fluconazole. Nanomedicine 5: 382-6.

18. Singh M, Kumar M, Kalaivani R, Manikandan S, Kumaraguru AK (2013) Metallic silver nanoparticle: a therapeutic agent in combination with antifungal drug against human fungal pathogen. Bioprocess Biosyst Eng 36: 407-15.

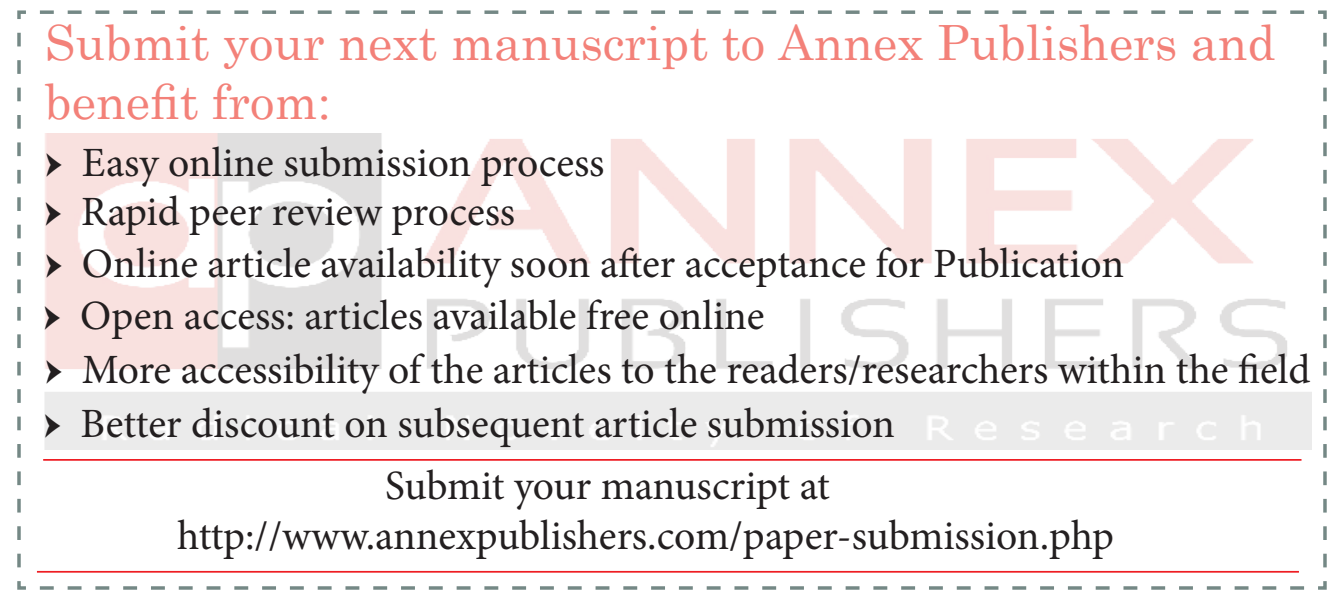

